CDD: 530.01

\title{
THE TRANSCENDENCE OF TIME IN THE EPISTEMOLOGY OF OBSERVATION FROM A PHENOMENOLOGICAL STANDPOINT
}

\author{
STATHIS LIVADAS
}

livadas@math.upatras.gr

Received: 28.07.2010; Accepted: 29.11.2010

\begin{abstract}
In this article I deal with time as a notion of epistemological content associated though with the notion of a subjective consciousness co-constitutive of physical reality. In this phenomenologically grounded approach I attempt to establish a 'metaphysical' aspect of time, within a strictly epistemological context, in the sense of an underlying absolute subjectivity which is non-objectifiable within objective temporality and thus non-susceptible of any ontological designation. My arguments stem, on the one hand, from a version of quantum-mechanical theory (History Projection Operator theory, HPO theory) in view of its formal treatment of two different aspects of time within a quantum context. The discrete, partial-ordering properties (the notions of before and after) and the dynamical-parameter properties reflected in the wave equations of motion. On the other hand, to strengthen my arguments for a transcendental factor of temporality, I attempt an interpretation of some relevant conclusions in the work of J. Eccles (1992) and of certain results of experimental research of S. Deahaene et al. (2005) and others.
\end{abstract}

Keywords: Absolute subjectivity. Decoherence functional. History filter. Phenomenological perception. Temporal consciousness. von Neumann's projection postulate.

\section{Introduction}

As it is implied by the title this article is an attempt to provide within an epistemological context a strong evidence towards a transcendental factor of temporality mostly as it is manifested in physical phenomena associated with interactions on the quantum level; the latter can be 
justified on the grounds that, generally, in a quantum system time is regarded as an external parameter partly because it can be taken as a co-constitutive factor in shaping the objective existence of quantum objects e.g. in quantum decoherence phenomena (see French (2002)). In view of my overall approach I chose to deal with a particular offspring of quantum measurement theory, the (consistent) time-histories theory, for the main reason that it is more focused than canonical quantum theory, both formally and interpretationally, on the role of time parameter in the context of quantum measurements. ${ }^{1}$ On this account it purportedly claims to a more rational comprehension of time in both its discrete and continuous aspects in the process of quantum measurements and a more consistent formal elaboration of it so as to provide an appropriate framework for ongoing theoretical activity on the subject.

There is a further epistemological dimension to the question of the transcendence of temporal consciousness engendered mainly by the works of J. Eccles (1992) and S. Dehaene et al. (2005) and also the works in Handy et al., (2003) and di Russo et al., 2005. The first is a quantum mechanical interpretation of the 'choice' between possible exocytosis states in the neuronal net of synapses which leads to the assumption of a mental subjectivity which reaches by means of psychons (or mental unities) the quantum exocytosis states in a unity-to-unity connection and it also demonstrates the reality of this effect as a livedin experience schematized by the interconnection between Worlds 1 and $2 .^{2}$

\footnotetext{
${ }^{1}$ In this view, canonical quantum theory is considered as conceding too much on the interpretational level to paradoxical credos such as the Copenhagen School's wavefunction collapse or to interpretationally evasive decoherence assumptions as von Neumann's projection postulate.

${ }^{2}$ J. Eccles (1992) schematically describes World 1 as the part of the brain that serves as an intermediary to World 2, which is referred to as comprising those mental components representing interior and exterior perceptions and also self-conscious mind; see Eccles (1992), pp. 272-273.
} 
Moreover Eccles' views can be seen as a sum up of corroborative evidences based in part on experimental data that lead to a notion of a subjective factor, termed by him spirit, acting as a unifying pole of lived experiences through a non-material field analogous to the probability fields of quantum mechanics (Eccles (1992), p. 253). In this respect his self-conscious spirit can be linked to the phenomenological notion of ego as an ever-in-act subjective factor making possible by its very objectification the unity of lived experiences as an unbreakable whole within the temporal flux of consciousness.

Concerning the work of S. Dehaene, I mainly emphasize his results in Dehaene et al. (2005) that present, as I will argue in a later section, a strong experimentally grounded claim for the relevance of a phenomenological approach towards a constitution of intentionally perceived objects as immanent objects of consciousness within a selfconstituting temporal substratum that can be, in fact, an object of reflective attitude solely by its own objectification. In the specific phenomenon of attentional blink, Dehaene presents some results that point to a difference between conscious and unconscious processing, on the one hand in the sense of a response triggered by events taken as primary correlates of conscious perception and on the other by an underlying unconscious process manifested in the detection of brain potentials which are not evoked by some kind of conscious activity.

The difficulties encountered in finding a linguistically meaningful way to deal with the absolute subjectivity behind objective temporality are brought up in Section 5 where a brief reference is made to Husserl's late work on temporality in Bernauer Manuskripte. There, as well as in his recently published last writings on temporality in Husserl (2006), he constantly moves from the Scylla of getting trapped into objective temporality to the Charybdis of generating a predicative discourse for what is by nature non-predicative. This is meant as a concluding section, inasmuch as it focuses on the core problematic of inner time 
consciousness coming out of a phenomenologically motivated review of the question of time in an epistemological context, as it was elaborated in the preceding sections.

\section{The subjective temporal substrate of a formal theory}

The main purpose of this section is to put forward certain arguments on the relevance of the phenomenological approach towards objects of formal theories, e.g. of quantum theory, which are abstracted from registered facts of physical reality through some kind (quantum mechanical in this case) observation. At the same time, the question of time and the source of temporality as inherently linked to the notion of constitution of well-defined objects of intentional observation will be thoroughly considered. In this respect, my approach is a phenomenologically motivated one in regarding, first, formal sciences within a context of intentionalities and second in regarding the apophantic domain of theoretical forms such as those introduced in Formale und Transcendentale Logik (FTL), Husserl (1974), as receiving their senses from the objective domain yet interpreting them as supposed (Sokolowski (1974), pp. 286-288). This point of view may generally lead to a new approach beyond that of pure logic and mathematics taken as diverse disciplines in classical tradition or may yet lead beyond an extensional approach producing paradoxes of the kind of Russell's antinomy.

This approach would seek a common ground underlying the objective and apophantic domain of a mathematical theory respectively shaped by the following principal fields: the field of formal ontology using categories referring to objects and the field of formal apophantics using categories referring to meanings, as they were mainly developed in FTL. As it will be elaborated in the following, both fields are re- 
duced on a still deeper level to a notion of object as an intentional object whose meaning is ultimately grounded on being the content of an intentional act in temporal fulfillment and also to the notion of an internal phenomenologically constituted time.

The aforementioned fields shape a judgement in clear and distinct existence in terms of the following: a) Once a judgement is brought out in full existence in distinction to any other it already refers to objects as noematical correlates of registered facts bestowed with the meanings which is all that is expressible about them ${ }^{3}$ e.g., the relations subject-predicates attached to it or subject of an act-object of an act in the synthetic unity of the three temporal states of judgement, (1) before, (2) during and (3) after registration (Sokolowski (1974), p. 240) and b) Any judgement at the level of distinction should be inevitably reduced, no matter how syntactically complex, to ultimate object-substrates (Husserl (1974), pp. 210-213) which are deprived of any analytical character being only capable of a sole qualification as phenomenological evidences.

The second condition is the core matter of formal ontology which deals with objects as registered intentionalities of a living subject, bearer moreover of a consciousness, with all categorial objectivities associated with them irrespective of being taken as irreducible 'thingness' substrates (sachhaltige Substrate) or 'empty' substrates in complete abstraction. These latter objects (or aggregates of them) are referred to in Ideas I as (last) empty substrates (Leersubstrate). Empty substrates as states of affairs (Sachverhalte) in complete abstraction deprived of any trait of material content (since they are not considered to be variations over common content) can be thought of as objects of mathematical logic in the form of numbers, or of elements of sets, of classes of sets,

\footnotetext{
${ }^{3}$ In Husserl's view in Ideas I (pp. 297-298) the content of an intentional act is thought of as the 'meaning' of the act by virtue of which consciousness refers to an object or state of affairs as its own.
} 
of domains of functions, etc (Husserl (1995), p. 33). Taken that apophantic sentences receive the sense of their objects from the domain of experience, albeit they interpret them as supposed, and that these objects are ultimately reduced to last object-substrates registered by hyletical-noetical moments of intentionality, we can put up the following claims based on the fact that these moments are not of a psychological character but rather something a priori grounded on the idea of knowledge as such and associated with some form of intentionality (Husserl (1975), p. 240).

One can soundly raise the possibility of forming an object of knowledge roughly based on the following conditions referred to in Logical Investigations: a) the a priori character of the noetical form of intentionality independently of any concrete empirical act conditioned on psychological constraints and b) the purely logical character of the ideal conditions of an object's knowledge grounded on the 'content' of the act of knowledge. On account of the first condition a thinking subject should be, in principle, capable of implementing all sorts of acts to ground theoretically his knowledge and on account of the second condition we should consider theoretical meaning, associated with truthfulness of judgements in descending order and with logical laws reducible to fundamental logical principles, as expressions of conditions grounded on the 'content' of acts of knowledge. Though these laws as a priori conditions of knowledge can be taken as such independently of a possible relation to a subjectivity, yet they were considered by Husserl as somehow 'susceptible to a reversal' by means of which they acquire as expressive experiences (ausdrückliche Erlebnisse) a relation towards a knowing subject.

This is also claimed in the supplementary volume to Logical Investigations completed in 1913, (Husserl (2002), where the expressibility of lived experiences is explicitly conditioned on the acts of judgement of a subject who, irrespective of whether an object of judgement is tran- 
scendental or immanent to his consciousness, he can form an expression referring, for instance, to an experienced feeling of desire, in fact any lived-in experience non-expressible as such, by a judgemental act that: directs to it a reflective intentional perception (Wahrnehmung), puts it under the general meaning of 'desire' and through this meaning and the particularity of the content of the specific desire gives it its definite meaning (Husserl (2002), p. 63). Husserl indirectly introduced at this stage an intentional subjectivity, associated with his well-known thematic from Ideas I and the Phenomenology of Inner Time-Consciousness on intentionally constituted objects of consciousness, by referring to the meaning of a word-object, taken as supposed within a linguistic form, as the reflective expression of the 'empty significative intention' corresponding to it (Husserl (2002), p. 74).

The matter is further clarified pertaining to the meaning of a theory (also of a truth) in view of their standing as the ideal contents of a possible knowledge. By this token, a single truth corresponds to the same content of a multiplicity of individual knowing acts put up then as their ideal and identical content. Inversely, to a multiplicity of individual knowing acts, by means of which each time and for any individual subject the same meaning becomes the content of a knowing act, corresponds the same meaning as their ideal identical content. Although Husserl claimed that in this way meaning is built up not on acts but on ideal elements such as truths and principles, e.g. the ideal form of premiss and implication, it is nevertheless conditioned on the capability of performing acts reducible to a priori noetical ones on the part of an (intentionally) oriented subject (Husserl (1975), pp. 240-242). This last claim constitutes, in fact, Husserl's turning point in Logical Investigations at least with regard to his previous psychologistic description of objects of arithmetic and algebraic theory in his Philosophy of Arithmetic. Furthermore, a common foundation of object and meaning was envisaged as the double-sided content of knowing acts in terms of which 
the possibility of a theoretical knowledge can have no other sense than the meaningfully thought objects; in reverse, as we can turn back from objects to meanings the possibility of a theory can 'mean nothing else than the "validity" or better the substantivity (Wesenhaftigkeit) of the related meaning.' (ibid. p. 242).

In this sense, talking about the meaning of an object and the fulfillment of a meaning-oriented intention essentially express the same thing inasmuch as objects are thought of as contents of intentional acts and their intuiting (Anschauung) as fulfilled through a meaning-oriented intention in a dynamical relation unfolded within phenomenological temporality (Husserl (2002), pp. 39-40). In what proves to be a fundamental difference between the constituting and the constituted level, Husserl considered the temporally constituted objects of an intentional act of cognition to be in a statical relation, whereas he considered the realization of any intentional act towards its content, inasmuch it is a fulfillment within temporal consciousness, to 'be' in a dynamical relation.

Overall, the solid foundation of meaningfully thought objects irrespective of whether they are taken as fundamentally registered by intentionality (formal-ontological objects) or as supposed (objects of an apophantic domain) is traced back to their possibility of existence as fulfillments of the intentionality of a temporal consciousness. It provides, in effect, a common view of registered-in, lowest-level intentional objects and their formal-ontological representations (including in abstraction objects of logical-mathematical theories) as being constituted as re-identifying immanences within temporal flux, which is, in turn, ultimately conditioned on the transcendental root of the unity of temporal consciousness. As I will try to show in the next sections this kind of transcendence within immanent temporality (referred to by Husserl as the absolute ego of consciousness or absolute subjectivity of the flux in Husserl (1966), p. 75) stands as a metaphysical 'vacuum' in the 
epistemological foundations of formally representable physical theories such as Quantum Mechanics. This might be relevant too, concerning abstract formal theories in the context of mathematical foundations and could be the field of further research.

\section{Temporality in the histories approach to quantum theory}

My main purpose in the following will be to show, taking into account that time enters in quantum theory as an external parameter, ${ }^{4}$ that there is not a formally definable way to capture the transition from a proposition referring to a 'sharp' moment of time to a proposition referring to the time-history of a quantum event. In other terms, that there is no way to 'capture the residuum' between a measurement at a 'sharp' moment of time and the subsequent spontaneous reflection on the particular measurement (in terms of the triangle: quantum objectmeasuring apparatus-conscious observer), without applying quantum principles such as the von Neumann-Lüders reduction postulate (applied also in the form of von Neumann's projection postulate in canonical quantum theory) or without formally assuming standard continuity and infinity in applying integral and differential calculus; or yet, alternatively, by applying the principles of non-standard mathematics which may be viewed as a non-conventional way to incorporate classical infinities and infinitesimalities within formal discourse.

\footnotetext{
${ }^{4}$ The main motivation in dealing with the question of temporality on the quantum level is the fact that the time parameter in quantum mechanical processes is regarded as external to the system thus susceptible to a phenomenologically motivated interpretation in evident difference with the notion of time as an internal parameter of macroscopic physical systems in newtonian or relativistic mechanics.
} 
I note in passing, that the application of nonstandard mathematical notions to quantum mechanical formulation can be seen as a way to capture, for instance, 'instantaneous' transition of states of dynamical observables in a way that no distinction is made between the continuous and discrete spectra of observables. Specifically, by defining an ultra eigenvector $f$ to be a unit vector $(\|f\|=1)$ corresponding to a unique eigenvalue $\lambda$ that belongs to the spectrum of an operator $A$ (irrespectively of whether it is discrete or continuous) such that $\|A f-\lambda f\|$ is infinitesimal, one can formulate the axiom of measurement of a nonstandard version of quantum mechanics like this: 'The result of any measurement of an observable can only be one of the standard spectral values of the corresponding operator. As a result of the measurement, the physical system finds itself in a state represented by an ultra eigenvector of the operator representing the measured observable, corresponding to the measured spectral value'. (Farrukh (1975), pp. 178-179 and p. 191). This is particularly relevant in the case of transition to states $\left\{g_{i} ; i \in I\right\}$ corresponding to the same ultra eigenvalue $\lambda$ as to that of the ultra eigenstate representing the operator $A$ after measurement by means of the relations: (i) $\left\|A g_{i}-\lambda g_{i}\right\| \approx 0 \forall i \in I$ (i.e. $\left\|A g_{i}-\lambda g_{i}\right\|$ infinitesimal) and (ii) (the non-standard form of the orthogonality condition) st $\left\langle g_{i}, g_{j}\right\rangle=\delta_{i}^{j} \forall i, j \in I$ where $\delta_{i}^{j}$ is the well-known symbol defined by: $\delta_{i}^{j}=1$ for $i=j$ and $\delta_{i}^{j}=0$ for $i \neq j$ (Farrukh (1975), p. 191). But this non-standard reformulation of quantum mechanics may finally reduce to ambiguities associated with the set-theoretical foundations of non-standard mathematics themselves, e.g. concerning the axiomatical treatment of non-standard magnitudes (see Livadas (2005)).

Further we may note, regarding a view of quantum individuality (the Space-Time Individuality, S-T.I.) in which it is the points of spacetime that confer both individuality and re-identifiability to quantum objects in the sense that there is nothing but the properties and the 
points of space-time at which they are instantiated (French (1989), p. 439), that a significant underlying factor in the description of a sequential quantum measurement lies in the texture of the topology of configuration space; for instance, in the construction of a space of history propositions in Lorentzian manifolds with a non-globally hyperbolic metric as it will be described in some detail later. However, this modelisation can be regarded, independently of the particular context, as a formal structure rooted ultimately in an idea of a 'pre-existing' subjective continuum.

In this context, I am rather inclined in the quantum histories approach to the notion of quantum measurement initiated by GellMann \& Hartle (see, Gell-Mann \& Hartle (1990a), Gell-Mann \& Hartle (1990b), Hartle (1993)) and subsequently by C. J. Isham as an alternative way to capture temporal transition in terms of sequential propositions corresponding to measurements, by applying a quantum version of temporal logic rather than the single-time logic of the singletime propositions approach of canonical quantum theory (see Isham (1993) and Isham (1994)). A key supposition, motivated by 'the problem of time' in a quantum gravity context, is that the familiar concepts of space-time 'emerge' well above the Planck scale as also the Hilbert space mathematical formalism of canonical quantum theory tied to the standard picture of space and time. As a matter of fact, a historiesbased interpretation of quantum theory inasmuch as it is associated with this particular non-standard version of the single-time propositions approach can be seen as moving from observables to 'beables' something that is particularly attractive in any theory attempting to address issues of quantum cosmology (Isham (1994), p. 2).

On this account, I point to the claim, (e.g. Savvidou (1999)), that the formalism of a (consistent) histories approach to quantum propositions that involve the time parameter, mathematically distinguishes between two qualities of time: 'its partial ordering properties (the no- 
tion of before and after) and its status as a dynamical parameter in the equations of motion' (Anastopoulos (2001), p. 3227). In this sense the consistent histories approach deals with sequences of values of quantities and not with results of measurements of quantities thus surpassing the dubious theoretical assumption of the 'collapse' of the state vector whenever a measurement is made in the standard interpretation of quantum theory. However, there are still problems arising from the definition of a locally defined notion of 'internal' time in quantum gravity and the ensuing physical repercussions associated with the particular choice of internal time. Under these assumptions the distinction on the formal level between the two forms of time ('being' in quantum temporal logic and 'becoming' in dynamical equations) looks questionable (Isham \& Savvidou (2002), pp. 23-24).

In general, it seems that the key to understanding the formal representation of time in theoretical physics may lie in a deeper comprehension of the double role of real numbers in labelling (ordering in strict mathematical sense) the points of 'being' and also pertaining to the 'becoming' in the application of the dynamical differential equations, e.g. Scrödinger's equation, which is mixing up in its standard form the two concepts of time (the discrete, stepwise and the continuous one). My point is that even in the consistent histories theory one may still get 'trapped' in the impredicativity of a kind of internal temporality reflected in the mathematical structure of temporal supports associated with consistent histories of propositions; i.e., reflected in the adoption of topological structures such as the basic regions (open sets) as nuclear temporal supports of a quasi-temporal situation (Isham (1993), pp. 29-30), a supposition that will be shown to presuppose the implicit assumption of the impredicative mathematical continuum.

In particular, the formal distinction between the two qualities of time in the consistent histories approach mentioned above is seen, for instance, in the definition of the probability assignment: 


$$
\begin{aligned}
& \operatorname{Prob}\left(\alpha_{t_{1}}=1 \text { at } t_{1} \text { and } \alpha_{t_{2}}=1 \text { at } t_{2} \text { and... } \alpha_{t_{n}}=1 \text { at } t_{n} ;\right. \\
& \left.\rho\left(t_{0}\right)\right)=\operatorname{tr}\left(\alpha_{t_{n}}\left(t_{n}\right) \ldots \alpha_{t_{1}}\left(t_{1}\right) \rho\left(t_{0}\right) \alpha_{t_{1}}\left(t_{1}\right) \ldots \alpha_{t_{n}}\left(t_{n}\right)\right) \quad(\mathbf{I})
\end{aligned}
$$

which is the joint probability of finding all the properties corresponding to a sequence of measurements corresponding to propositions $\alpha_{t_{1}}, \alpha_{t_{2}}$, $\ldots, \alpha_{t_{n}}$ at times $t_{1}, t_{2}, \ldots, t_{n}$, and in the definition of the decoherence functional $d(\alpha, \beta)=\operatorname{tr}\left(\widehat{C}_{\alpha}^{+} \widehat{\rho}_{0} \widehat{C}_{\beta}\right)$ whose properties determine the satisfaction of conditions rendering the probability assignment (I) meaningful even for a closed system (Isham (1993), p. 7); that is, even in the absence of of an external 'observer' with associated measurementinduced state vector reductions. ${ }^{5}$ The remarks below concern the class operator

$$
\widehat{C}_{\alpha}=\widehat{U}^{+}\left(t_{1}\right) \widehat{\alpha}_{t_{1}} \widehat{U}\left(t_{1}\right) \ldots \widehat{U}^{+}\left(t_{n}\right) \widehat{\alpha}_{t_{n}} \widehat{U}\left(t_{n}\right)
$$

$(\widehat{U}(s)=\exp (-i \widehat{H} s)$ is the unitary time-evolution operator $)$ that represents a history $\alpha$ in a discrete-step causal evolution corresponding to a string of projection operators $\widehat{\alpha}_{t_{1}}, \widehat{\alpha}_{t_{2}}, \ldots \widehat{\alpha}_{t_{n}}$, and also the projection operators corresponding to a homogenous history $\alpha^{6}$ in probability assignment (I); they are also relevant in the general case of HPO (History Projector Operator) theory, referring to a complete space $\mathcal{U P}$ of history propositions, where a quasi-temporal type of dynamical evolution

\footnotetext{
${ }^{5}$ The decoherence functional $d(\alpha, \beta)$ is a complex-valued function of a pair of histories $\alpha, \beta$ that measures their mutual quantum interference (being indirectly a probability measure) such that a set of exclusive and exhaustive histories is called consistent if for all pairs of different histories $\alpha, \beta$ in this set the equation $d(\alpha, \beta)=0$ holds.

${ }^{6} \mathrm{~A}$ homogenous history is defined as any time-ordered sequence $\left(\widehat{\alpha}_{t_{1}}, \widehat{\alpha}_{t_{2}}, \ldots, \widehat{\alpha}_{t_{n}}\right)$ of projection operators.
} 
together with an associated Heisenberg picture can be implemented (Isham (1993), p. 24).

Thus in Def. (II) we have in the same formula two completely different aspects of time parameter, the discrete part linked to a sequential order of temporal steps and the continuous part incorporated as an argument of the exponential form of time-evolution operator $\widehat{U}(s)$, in the sense that the operator $\widehat{C}_{\alpha}$ depends also explicitly on the dynamics of the system as it uses the Heisenberg-picture operators $\widehat{\alpha}_{t_{n}}\left(t_{n}\right)$. This means that the aspect of temporal order represented by the string $\widehat{\alpha}_{t_{1}}, \widehat{\alpha}_{t_{2}}, \ldots, \widehat{\alpha}_{t_{n}}$ of projection operators that represent history $\alpha$ (formally defined in Isham (1993) as a history filter $\alpha$ to provide a formalization of it as a sequential conjunction defined as 'active' over a finite set of temporal points) is conditioned on the assumption of a continuous function $\widehat{U}(s)=\exp (-i \widehat{H} s)$ representing the dynamical evolution of the system with definite values $\widehat{U}\left(t_{1}\right), \widehat{U}\left(t_{2}\right), \ldots, \widehat{U}\left(t_{n}\right)$ at corresponding time steps $t_{1}, t_{2}, \ldots, t_{n}$. The dynamical part of the class operator $\widehat{C}_{\alpha}$ generates moreover the transformations $\widehat{\alpha}_{t_{1}} \rightarrow \widehat{\alpha}_{t_{2}}$ in the continuum limit.

Thus, in the histories formalism the sequential conjunction of properties in finitely many ordered time steps cannot be effected but on the underlying assumption of time as the parameter of a Heisenberg-type evolution which means that one is left with a residuum of a continuous time-flux formalized in terms of the continuous argument of unitary time-evolution operator.

In fact, as it will be made clear next, my overall argument is also indirectly connected with the construction of a new Hilbert space $\otimes_{t \in \mathcal{T}}^{\Omega} H_{\mathcal{T}}$ associated with an infinite tensor product $\otimes_{t \in \mathcal{T}}^{\Omega} B(H)_{\mathcal{T}}$ of operator algebras $B(H)$ to accommodate arbitrary temporal supports. The tensor product $\otimes_{t \in \mathcal{T}}^{\Omega} B(H)_{\mathcal{T}}$ is defined to be the weak closure (in the weak operator topology) of the set of all functions from $\mathcal{T}$ to $B(H)$ that are equal to the unit operator for all but a finite set of $t$-values 
(Isham (1993), p. 23). This topological property ensures, in fact, that any history, even one corresponding to an infinite set of projection operators, can be formally treated as the weak limit of a convergent sequence of homogenous histories with finite (temporal) support. ${ }^{7}$ Indeed, the overall formal structure of $\otimes_{t \in \mathcal{T}}^{\Omega} H_{\mathcal{T}}$ is such that it is fundamentally connected with a notion of temporal support defined as the finite set of time-points $t \in \mathcal{T}$ for which a history proposition $\alpha_{t} \neq 1$; that is, it is associated with the set of time-points for which the history filter $\alpha$ is the non-trivial proposition, in other words, it is associated with the finite set of time-points for which $\alpha$ is active (Isham (1993), p. 23).

The intrinsic need to refer to a finitistic temporal support on which to implement a sequential conjunction of the type ' $\alpha_{t_{1}}$ is true at time $t_{1}$, and then $\alpha_{t_{2}}$ is true at time $t_{2}$, and then ....and then $\alpha_{t_{n}}$ is true at time $t_{n}$ ' corresponding to a history filter $\alpha=\left(\alpha_{t_{1}}, \alpha_{t_{2}}, \ldots, \alpha_{t_{n}}\right)$ in the language of temporal logic can be noted, also, in the general situation where the HPO theory is applied to the quasi-temporal situation of a four-dimensional manifold $\mathcal{M}$ with a non-globally hyperbolic Lorentzian metric $\gamma$. In the particular case, the temporal support of the test function $f_{i}$ on $\mathcal{M}$ of each member $P\left(f_{i}, I_{i}\right), I_{i} \subset R, i=1,2, \ldots, n$, of a class of propositions corresponding to a history filter, is a finite collection of open subsets $O_{i}$ of $\mathcal{M}$ (termed basic regions $O_{i}$ ) which are topologically connected and have compact closure so that each proposition is localized in the space-time $\mathcal{M}$ and they are moreover

${ }^{7}$ The temporal support of a history filter $\alpha$ may be described as the finite set of time points $t \in \mathcal{T}$ for which $\alpha$ is active, i.e. those points $t \in \mathcal{T}$ such that $\alpha_{t} \neq 1$. Its underlying role is associated with the semi-group homomorphism $\sigma: \mathcal{U} \rightarrow S$ that assigns a temporal support $s \in S$ to each history filter $\alpha \in \mathcal{U}$ in such a way that a history filter $\beta=\left(\beta_{t_{1}^{\prime}}, \beta_{t_{2}^{\prime}}, \ldots, \beta_{t_{m}^{\prime}}\right)$ is said to follow a history filter $\alpha=\left(\alpha_{t_{1}}, \alpha_{t_{2}}, \ldots, \alpha_{t_{n}}\right)$ if $t_{n}<t_{1^{\prime}}$ and the combined history $\alpha \circ \beta$ is defined as $\alpha \circ \beta=\left(\alpha_{t_{1}}, \alpha_{t_{2}}, \ldots, \alpha_{t_{n}}, \beta_{t_{1}^{\prime}}, \beta_{t_{2}^{\prime}}, \ldots, \beta_{t_{m}^{\prime}}\right)$. This means that, by virtue of the homomorphism equality $\sigma(\alpha \circ \beta)=\sigma(\alpha) \circ \sigma(\beta)$ any sequential conjunction of projection operators corresponding to a history proposition is 'tied' to a (causally evolving) ordered temporal conjunction. 
associated in such a way that any two $O_{i}$ 's either follow one another or they are space-like separated in alternative case (Isham (1993), p. 30). This assumption which underlies the definition of a 'time point' as a finite collection of disjoint basic regions space-like separated from each other leads to the following argument: the individuality of each time-point, inasmuch as it is associated with the realization of a proposition $P\left(f_{i}, I_{i}\right)$, is grounded on its definition as a nuclear support $s$ under semi-group composition law o, i.e., it cannot be written in the form $s=s_{1} \circ s_{2}$ where both constituent nuclear supports $s_{1}, s_{2}$ are different from the unit (temporal) support $*$; in other words, individuality of each 'time-point' is associated with a unique and irreducible intentional act in the sense of 'performance' of an act of quantum measurement inherently irreducible to any class of causally related acts. In this sense, in general history theory, a nuclear support is meant as analogous to a time-point abstracted as an extensionless point of the real continuum (think of a natural number within real continuum) in terms of irreducibility to any further temporal subdivisions.

However, the 'finitistic' character of an act of reflection upon a particular intentional act of measurement carried out in the present now of the flux of consciousness is represented by the topological properties of compactness and connectedness assigned to each of the finitely many basic regions $O_{i}$ of a nuclear support $s$ while letting all the same slip a 'creeping' continuity factor by the topological definition of each basic region $O_{i}$. Thus in the quasi-temporal logic associated with a non-globally defined temporality the impossibility to associate a single-time proposition with a physical measurement enacted in a fictitious 'dimensionless' time-point is reflected in the formal definition of the nuclear support $s$ as set-theoretically non-point-like while underscoring, at the same time, on the formal level the deficiencies engendered by the definition of time-points as atemporal abstractions in formal representation. Moreover from a certain viewpoint, the afore- 
mentioned definition of a nuclear support may be associated with the Husserlian notion of specious present as a non-point-like present now of original impression apriorically (in objective temporal continuity) connected with athematic protention (a kind of expectation) and fulfilled retention (a kind of memoration) while retaining the 'finitistic' character of an instantaneity in objective reflection (see, Husserl (1966), pp. 29-31 and pp. 52-53). Of course such an assertion would imply, in the first place, a view of mathematical points, taken here as finite classes of space-like separated open sets equipped with a compact and connected topological structure, as complete abstractions by categorical intuition of empty forms of thingness-substrates on the lowest level of intentional perception (p. 439); second, it would condition their status as formal-ontological objects on their prior phenomenological foundation as re-identifying temporal individual-substrates.

On the other hand, there is an acceptance of a version of the von Neumann projection postulate in deriving the sequential conjunction of propositions of the form ' $\alpha_{t_{1}}$ is true at time $t_{1}$ and then $\alpha_{t_{2}}$ is true at time $t_{2}$ and then.... $\alpha_{t_{n}}$ is true at time $t_{n}$ ' contained in the definitions of joint probability and the decoherence functional of histories. In definition (I), for instance, the following formula for the density operator state, retained for further calculations, is valid by von Neumann-Lüders reduction:

$$
\rho_{\text {red }}\left(t_{1}\right):=\frac{P\left(t_{1}\right) \rho\left(t_{0}\right) P\left(t_{1}\right)}{\operatorname{tr}\left(P\left(t_{1}\right) \rho\left(t_{0}\right)\right)}
$$

where $\rho\left(t_{0}\right)$ is the density operator state at time $t_{0}$.

In my view, the implicit application of the above version of von Neumann's projection postulate in the histories formalism, conditions the derivation of the joint probability $P$ and the decoherence functional $d(\alpha, \beta)$ on the possibility of a joint assignment of values for history propositions on singleton-set temporal supports $\left\{t_{i}\right\}$ upon an underlying subjective temporal continuity; this can be further associated with 
an assumption of existence of a subjective continuous temporal unity on which to constitute objects of intentional perception and then apply a reflective regard on these constituted objects at once, as immanent objects within the homogenous flux. On this account, J. von Neumann's projection postulate can be seen as introducing indirectly the necessity for a self-constituting time flux by assigning to the (mathematical translation of) state $s(t)$ of a quantum quantity $Q_{i}$ upon a first-kind measurement ${ }^{8}$ that yields an eigenvalue $r_{\kappa}$ (within an interval $\left.r_{\kappa} \pm \epsilon_{Q_{i}}\right)$, the same eigenvector $\psi_{\kappa}$ as to the state $s\left(t_{1}\right)$ of the quantity $Q_{i}$ at time $t_{1}>t$ soon after the measurement. Even in the insertion of the trivial unit-proposition as the limit $\alpha_{t_{i}} \rightarrow 1$ within a homogenous history $\left(\alpha_{1}, \alpha_{2}, \ldots \alpha_{t_{i-1}}, \alpha_{t_{i}}, \alpha_{t_{i+1}}, \ldots \alpha_{t_{n}}\right)$, in the application of $K$-operators for which the evolution property

$$
K\left(t_{i-1}, t_{i}\right) K\left(t_{i}, t_{i+1}\right)=K\left(t_{i-1}, t_{i+1}\right)
$$

holds, a sort of retention of temporal values of transition operators must be assumed analogous to the conditions set by von-Neumann's projection postulate. As a matter of fact, assumptions of this type 'are incorporated' in the continuity of decoherence functionals on the set of all history propositions $\mathcal{U P}$ as dynamical information encoded in non-standard decoherence functionals via evolution operators $K$ of the above form. These operators act on Hilbert spaces $H$ associated with single-time propositions, within systems of a quasi-temporal structure (Isham (1994), pp. 33-34).

\footnotetext{
${ }^{8}$ This is one in which the measured system described by quantum state $s$ needs to interact with the measuring apparatus described by quantum state $\phi$, so that the total wave function before the interaction is $s \cdot \phi$ and by unitary evolution the final total wave function gets: $s \cdot \phi \rightarrow \sum_{n} c_{n} s_{n} \phi_{n}$ where $s_{n}$ are eigenstates of the operator to be measured and $\phi_{n}$ the orthonormal states of the measuring apparatus.
}

Manuscrito - Rev. Int. Fil., Campinas, v. 34, n. 2, pp. 435-469, jul.-dez. 2011. 
In the bottom line, definitions of a decoherence functional for continuous-time histories fail to provide a satisfactory definition of it as the inductive limit of the decoherence functional defined on $H^{I} \times H^{I^{\prime}}$ for all choices of finite partitions $I$ and $I^{\prime}$ of the time interval $T$ (taken as a subset of real line) for the formal reason that one cannot continuously embed the lattice of single-time propositions to the lattice of history ones. This problem is essentially due to the 'undefinability' of a sharp moment of time, abstracted as a point on the real axis of zero measure, since at this time-point an observable can be well-defined only if we take the function $f: T \rightarrow R,(T \subseteq R, R$ the set of reals) in the definition of time-averaged operators $A_{f}$, to be a delta function. But this is unacceptable in a consistent histories construction due to the fact that we have to define a decoherence functional for continuous-time histories as the inductive limit of a discrete-time expression defined on $H^{I} \times H^{I^{\prime}}$ for all choices of time-discretizations $I$ and $I^{\prime}$, whereas a delta function is known to run wildly at 'sharp' points.

Taking into account that attempts to properly define a continuoustime decoherence functional encounter various difficulties fundamentally associated with the incompatibility of the notion of temporal continuum on the one hand and that of a sharp moment in time on the other, the discussion about a decoherence functional associated in final count with a properly defined probability measure in a consistent histories approach may reduce to the following assertion: no matter the particular mathematical techniques employed to circumvent the prob$\mathrm{lem}^{9}$ the deeper issue is that we cannot, in fact, continuously embed the

\footnotetext{
${ }^{9}$ Certain alternative approaches such as incorporating the information about initial condition in an object that is extended in time or defining the decoherence functional with respect to a structure of propositions about phase-space histories that involve the mathematical notion of measurability, cannot be implemented without some unwelcome effects such as sacrificing the quantum logic structure of history propositions (see, Anastopoulos (2001), p. 3256).
}

Manuscrito - Rev. Int. Fil., Campinas, v. 34, n. 2, pp. 435-469, jul.-dez. 2011. 
lattice of single-time propositions to the lattice of history ones. This is a question that seems to run deeper into the character and inner modes of temporal constitution as a self-constituting continuous objectivity (in phenomenological terms) and may be reasonably taken to be reflected in formal metatheory in this kind of incongruity.

Before closing this section it seems worthwhile to consider for a moment the role of the aforementioned delta function $\delta(x)$ within quantum formalism. The essential reason for employing the concept of delta function is to provide a proper mathematical tool for treating certain kinds of infinities arising in mathematical formalism e.g. in P. Dirac's definition of the non-vanishing product $\langle X \mid Y\rangle$ of two kets expressed as an integral of eigenkets of an observable $\xi:\langle X \mid Y\rangle=\iint\left\langle\xi^{\prime} x \mid \xi^{\prime \prime} y\right\rangle$ $d \xi^{\prime} d \xi^{\prime \prime}$ (see Dirac (1981), p. $39 \&$ pp. 58-62). It is well-known then, that the function $\delta(x)$ such that $\int_{-\infty}^{\infty} \delta(x) d x=1$ and $\delta(x)=0$ for $x \neq 0$ is, in fact, not a function of the variable $x$ in the usual definition of a function, that is, as having a definite and unique value for each point in its domain but rather a mathematical convenience to ensure well-defined integrals in case wild variations of quantum variables bordering to infinity are involved. For that reason, it looks pointless to treat the delicate question of properly defining an observable at a 'sharp' moment of time by applying the delta function.

Maybe the whole issue of assigning a well-defined meaning to quantum properties and eventually to quantum probabilities in sequential time-measurements without getting trapped in the constraints of contextuality, as Bell's, Wigner's and Kochen-Specker's theorems demonstrate, reduces, to a significant extent, to a deeper understanding of time as a subjective constituting factor in shaping propositions about quantum properties. 


\section{Towards an epistemological corrobora- tion of the ego of absolute consciousness}

My main scope in this section is to put up some epistemologically motivated claims on the possibility of a transcendental root of the homogenous unity of temporal consciousness. As it was mentioned in section 1, I mostly rely on J. Eccles' and S. Dehaene's approaches in Eccles (1992) and Dehaene et al. (2005) to point out the relevance of the phenomenological analysis of temporal constitution, (see, Husserl (1966) and Husserl (2001)), taking into account their respective attempts to provide an epistemological context to the clues of an underlying presence of a subjective unifying ego of consciousness and, on the basis of experimental data, to point to a creeping unconscious level of 'awareness' even in the absence of any event-provoked activation of consciousness.

Concerning Eccles' view with regard to the notion of a unifying subjectivity termed as ego or spirit in Eccles (1992), I regard some of his claims not only susceptible of a phenomenological reading but moreover better formulated within a context incorporating an intentionally oriented subject who is also a bearer of a self-constituting temporal consciousness in terms of which he can 'transpose' the non-predicative evidence of his experience. In view of this, it is remarkable that Eccles gives a temporal dimension to the underlying 'factor' of mental unity by pointing to a subjective apprehension of everyone's mental unity as manifested in the continuity of most distant memories; in fact, this dimension is proclaimed as the base of ego, in the sense that it provides at each moment the unity of experience in reflection by integrating an almost infinite number of neuronal activities taking place in the brain at the same moment. In view of this kind of subjectivity Eccles makes reference to W.R. Uttal (1978), who has arrived on operational terms at a 'metaphysical' description of spirit as being of a holistic and non- 
divisible nature which is a problem utterly unresolved by psychology; or yet to K. Lorenz in (1977), who alludes 'to the mysterious barrier which is in the center of what constitutes the unity of our personality and separates the objective physiological events taking part in our body from the subjective experience we have of them.' (Eccles (1992), p. 272).

Moreover, he is inclined to assert that a self-conscious spirit is not only a 'passive onlooker' of the neuronal activities of the brain taking place all at once in front of its 'regard' but it is enacting its intentional regard to choose among an infinite plurality of such activities at any instant in any zone of the brain (Eccles (1992), pp. 273-274). This way the self-conscious spirit unifies the living experience in a way analogous to the act of phenomenological ego which constitutes the immanent flux of events by constituting itself. But in attempting to describe this unifying activity of spirit in terms of a type of quantum mechanical interaction with the brain he falls, as I will try to show next, in the closed loop of producing new transcendences in the place of those associated with the holistic, non-material nature of self-conscious spirit. This may be taken as corroborating my main claim in this paper, namely, that there is indeed a hidden immanent transcendence in the constitution of each one's temporal consciousness which is, in fact, the subjective everin-act constituting factor of temporal unity; this is utterly not possible to be predicated as existing according to the descriptive norms of the language of a formal metatheory but only 'after' its own objectification as an impredicative, continuous substratum.

As a matter of fact, J. Eccles proposed an epistemologically based interpretation of the interaction spirit/brain by assuming that this interaction is analogous to a field of probability in quantum mechanical theory which does not possess mass or energy but can nevertheless cause tangible effects in a micro-site. By this assumption a reflection on an intentional act can provoke neuronal effects in a way parallel 
to the probability fields of quantum mechanics (Eccles (1992), p. 253). Accordingly, one may adopt a model based on the transmission of cellular activities, that is, the structure of neuronal synapses associated with transmission by exocytosis of presynaptic vesicles ${ }^{10}$ which are indeed of an order of magnitude susceptible to the application of Heisenberg's Principle of Uncertainty ${ }^{11}$. In this model the phenomenon of exocytosis of a vesicle through the synaptic membrane can be effected by an intentional act acting in the sense of a field of quantum probability.

To produce macro-effects capable of a modification of neuronal activity, Eccles proposes to consider as neuronal unity a structure called dendron, composed of a bundle of dendrites and comprising tens of thousands of presynaptic vesicular nets susceptible to be 'chosen' for exocytosis; each dendron can be penetrated by a mental unity termed psychon on a unity-to-unity, non-local relation. These mental unities (psychons) are taken to serve as intermediaries in the bidirectional relation spirit/brain representing in particular and uniquely on the physiological level the dendron on which they are 'attached'; nonetheless they are left with no further specification as to their epistemological or even ontological status and no less with a dubious temporality. This remark can be drawn by their presumed role in channeling non-locally a mental intention to tens of thousands of activated presynaptic vesicular nets through each dendron and in reverse order in registering the effect of each vesicular exocytosis and transmitting it to the mental World 2 which in Eccles's classification comprises internal and external perceptions and also the interacting self-conscious spirit (Eccles (1992), p. 273). The acts of psychons should take effect, though, in objective time for it could be otherwise impossible to conceive them as a physical interaction and consequently even if we would take them as some kind

\footnotetext{
${ }^{10}$ Roughly speaking, presynaptic vesicles are molecular agglomerates in the vicinity of neuronal synapses.

${ }^{11}$ See Eccles (1992), p. 255 and p. 249 for schematic diagrams of neuronal synaptic activity in the stages of interaction spirit/brain.
}

Manuscrito - Rev. Int. Fil., Campinas, v. 34, n. 2, pp. 435-469, jul.-dez. 2011. 
of non-material unities they should be necessarily temporal objectivities susceptible, in principle, to the eventuality of non-homogeneities of the spatio-temporality of the physical world. For that reason they can be hardly taken as a kind of 'atemporal messengers' of a constituting homogenous temporality. In such a case they should be regarded themselves as the self-conscious spirit and the constituting factor of temporality but then a circularity would be engendered as they should be by necessity assigned another interposing temporal objectivity to transmit mental intention as a physical world effect.

In view of my general approach, we should take into account, considering Eccles' description of the kind of transcendental subjectivity, termed a self-conscious spirit, the aforementioned circularity concerning the role of psychons in 'instantaneously' transmitting mental intentions to the extent that: it can be plausibly claimed that it provides us with clues to the transcendental source of subjective temporality inasmuch as it engenders a possibly endless regression of transcendences reached by making use of a physicalistic language (a quantum mechanical in the particular case) to describe its temporal objectification.

On the other hand, I find of a particular relevance with regard to a phenomenological approach, Eccle's claim in Eccles (1989) that a number of indications point to a relation of unity-to-unity concerning the act of each psychon upon its particular dendron as each unique, registered-in-actuality experience may be associated with a particular psychon. Inasmuch as this bidirectional 'interaction' is uniquely constituted by the presence of self-conscious ego and its intentionallike orientation towards a (possibly abstract) something, it can be rightfully taken close to intentional perception meant as as uniquely founded on lowest-level intentionality towards individuals. To the extent that 'lowest-level' individuals are themselves irreducible as original 
givennesses, they ground by this very token the uniqueness of each intentional perception towards a state-of-things (Sachverhalt) (Husserl (1974), pp. 212-214).

We may also establish a connection with a temporal unity constituting ego and also consider certain intentionality associated neuronal mechanisms in view of recent experimental evidence in the work of S. Dehaene et al. (2005), T.C. Handy's et al. (2003) and J.J. McDonald's et al. in di Russo et al. (2005), among others. Concerning, in particular, S. Dehaene's presentation in (2005) a phenomenological interpretation can be relevant inasmuch as: it establishes a difference between conscious and unconscious processing by detecting early intact potentials (P1 and N1) evoked by unseen words, which is a fact suggesting that these brain events are not primary correlates of conscious perception something that could be taken as grounded on a preexisting immanent process of consciousness prior to the 'enactment' of an intentional relation subject-object. In this sense the detection of 'pre-activation' potentials of the brain may indeed point towards the evidence of an existing, ever-in act, self-constituting temporal flux.

On the other hand, the detection of a rapid divergence around 270 ms after which brain events were evoked solely by seen words ${ }^{12}$ can

\footnotetext{
${ }^{12}$ In a repeated trials experiment, subjects were asked to respond to two visual targets $T_{1}$ and $T_{2}$ depicting number words during the attentional blink caused by the attentional demands of one, let's say $T_{1}$, of the tasks. In the dual task of responding to words on both $T_{1}$ and $T_{2}$ for short stimulus onset asynchrony (SOA), identification of the first target $T_{1}$ hinders the detection of the second target $T_{2}$, although $T_{2}$ is easily seen when no task on $T_{1}$ is required. Although no significant difference was observed in the early visual $P_{1}$ and $N_{1}$ waves (96 and $180 \mathrm{~ms}$ ) evoked by seen and unseen $T_{2}$ 's either in amplitude or in topography, a first difference was observed around 170 ms with a slightly stronger positivity for seen $T_{2}$ 's. A larger divergence was observed around $270 \mathrm{~ms}$ when seen $T_{2}$ 's evoked a stronger left-lateralized posterior negativity $N_{2}$ followed by a more anterior negativity $\left(N_{3}, 300 \mathrm{~ms}\right)$ that was absent for unseen $T_{2}$ 's. Two subsequent waveforms $\left(P_{3 a}, 436 \mathrm{~ms}\right.$, $\left.P_{3 b}, 576 \mathrm{~ms}\right)$ were also detected only when $T_{2}$ was seen; see, Dehaene et al.
} 
serve in corroborating the claim that the 'enactment' of intentional perception towards a particular object standing as an unambiguous and original givenness in front of the intentionality of consciousness together with the possibility of reflection upon this 'enactment' are $a$ priori capacities of a bodily consciousness radically different from the (transcendental) root of its self-constituting objective unity. Moreover, by virtue of the hyletical-noetical character of intentional perception one should expect an evidence of its 'enactment' in spatiotemporal terms which is, in fact, detected in present case by the triggering of a late wave of activation that is distributed through a network of cortical association areas. Moreover, the divergence between seen and unseen $T_{2}$ 's at $270-300 \mathrm{~ms}$ was detected as coinciding with the end of the late $P_{3 b}$ waveforms evoked by the $T_{1}$ task; a fact that, taken into account that the $T_{1}$ task affects components of event-related potentials (ERP) similar to those correlating with conscious access to $T_{2}$, lends support to the idea that these components of ERP index a capacitylimited stage capable of processing only one task at a time (Dehaene et al. (2005), pp. 1393-1396). There is yet a question here, analogous to the question of 'temporal residuum' discussed in Section 3, concerning a lingering temporality bridging in effect the detection of activation potentials evoked solely by seen words and the 'enactment' of conscious perception whose primary correlates are precisely these words. In other words, the temporal factor bridging the subjective awareness ratings concerning targets $T_{1}$ and $T_{2}$, taken in the sense of purely intentional objects, and the objective identification and "naming' of corresponding target-words in the sense of a reflection upon intentionalities. This empirical evidence might be again interpreted by the existence of an objectified absolute flux of consciousness in whose temporal homogeneity are 'embedded' by certain noematical modes the objects of intentional perception (Wahrnehmung).

(2005), p. 1392 .

Manuscrito - Rev. Int. Fil., Campinas, v. 34, n. 2, pp. 435-469, jul.-dez. 2011. 
In connection with T.C. Handy et al. experimental data in (2003) I point to the detection of certain visuomotor transformations by eventrelated potentials and by event-related $f M R I$ techniques facilitated by the presence of objects automatically grabbing visual-spatial attention. It must be noted that visually guided grasping movements are conditioned on a rapid transformation of visual representations into objectdirected motor programs. On this account, visual-spatial attention to event-related object locations was automatically activating cortex areas associated with visually guided actions and their planning. This activity might be associated with a notion of intentionally 'triggered' kinesthetical sensations in the sense described in the Husserlian texts of Ideas III (Husserl (1997), pp. 120-123).

Lastly, in connection with J.J.Mc Donald's et al. work in di Russo et al. (2005), I point to the evidence of an intentionally based visual time-ordered perception by means of the detection of attentional shifts provoked by a sudden sound stimulus in terms of enhancement of the amplitude of neural activity in visual cortex rather than in terms of processing speed in the earlier visual-cortical pathways.

\section{A phenomenological view of the character of temporality}

In the Bernauer Manuskripte, Husserl attempted to clarify the notion of the absolute ego of consciousness which was left rather vague and unrefined in the Phenomenology of Inner Time-Consciousness, (Husserl (1966)). As a matter of fact, in spite of the brave intellectual quest to elucidate the origins of temporality within the immanence of consciousness he was further led to the intricacies of the deeply transcendental character of the source of temporality. 
In these later texts, instead of the transcendental (or absolute) ego of consciousness of earlier texts he used various other terms essentially designating the same notion such as Substrate (Hintergrund), Original Process (Urprozess), Original-Living (Urleben) or yet Original LivingSelf (ursprüngliches Ichleben) while, nevertheless, not reaching an unambiguous elucidation of the deeper meaning of this notion meant as the constituting subjectivity of temporal unity. At one point he reached the conclusion that Urleben can neither be temporal itself nor can it make temporal objectivities within it perceived as such (Husserl (2001), p. 196). For, in case Urleben would be temporal we could then turn our 'reflective regard' towards the givenness of its phases which would be a temporal continuum in the scheme original impression in the present now - descending sequence into the past and thus the experience of these phases as givennesses themselves would be also a temporal sequence upon which to turn anew our reflection which would become again givennesses upon which to turn our reflection and this way in infinitum. In such a case we would end up in an endless regression of reflections meant as consciousness-of something in the sense of temporal acts extended in an infinite series of successive terms. What we should derive, in any case, is that evidently what becomes an object of reflection has to be in a temporal form and should be also taken to be identically the same in the flux of multiplicities of its givennesses.

In that case, how could we possibly take Original Process (or living) (Urprozess) to 'be' as a subjectivity-preceding reflection? Husserl suggested that in this case we would have a 'primary current' (Urstrom) perceived as a temporal current but which nevertheless cannot be consciousness of a temporal current nor a phenomenological perception of it (Husserl (2001), p. 197). This is meant in the sense that as Original Process steadily constitutes first-degree phenomenological time, it 'is' ever a consciousness process, a process of intentional experiences. For in the alternative case, it would be a process of original hyleti- 
cal data sufficient for the constitution of hyletical unities-of experience which imply, in turn, a constitution of durating immanent apprehensions (Auffassungen) assumed though not to be necessary in that case. It turns out that we know about Original Process through phenomenological perception and that it is plainly given as a process by means of a self-constituting consciousness that constitutes temporal objectivity. Put in other words: taking the evidence of an immanent event, we can accomplish a reflection upon its phenomenological perception, as well as we can reflect upon the flux of the parts and phases of its immanent temporal objectivity, upon the flux of 'experiences' which are themselves 'consciousness-of' of certain other experiences and upon all that pertains to them (Husserl (2001), p. 204).

In attempting to thoroughly comprehend the nature of Original Process, Husserl proposed to consider phenomenological time and events taking place within it as given by phenomenological perception. In this view, an event takes place within the unity of phenomenological time without its particular unity having by necessity the 'privilege' of being brought to reflection by means of phenomenological perception (Husserl (2001), p. 198). This remark motivates a view of Original Process as associated with a phenomenological perception of events prior to their immanent seizure (Erfassung) in the constituting temporality thus avoiding the assumption of an endless regression of reflecting subjectivities. In this connection he wondered whether the field of constituted temporality is the all-inclusive field of Original Living-Self, that is, whether Original Living-Self is a perception in the sense of an apprehensive (aufmerkendes) or properly apprehensive (sonderbemerkendes) seizure or rather a perception in broadest sense that is directed towards an intentional object without the object in question being taken as identical with the 'act', that is, with the intentional experience as consciousness of it (Husserl (2001), p. 199). 
Further, taking account that a known process would be itself an immanent event, how could it be that such a process might constitute another immanent event? In the last count, genetically talking (Husserl gets here deeper entrenched in a problematic bordering to metaphysics), would it be thinkable that all temporality for a conscious subject is there by means of a genetically emerging apperception (Apperzeption) where the unknown processes passing through are not temporality-constituting and are themselves in no way temporally constituted (Husserl (2001), p. 200). But as Husserl follows by grounding each hyletical-noetical act, each seizure in immanence manifested through the consciousness of their succeeding and durating objectivities, to unknown processes possible on the grounds of their apperception, he seems to get already trapped in the circularity induced at least by the linguistic connotation attributed to the term apperception. For how can we perceive a sequence even of genetically emerging apperceptions without reflecting on them in succession within temporality, something that presupposes the occurrence of unknown processes prior to these apperceptions and not as a joint 'consequence' of them?

Either in taking Original Process as a constant constituted-fromwithin temporality bearing time-fulfilled immanent objects upon which we can always draw a reflective glance or taking it as a 'current' with no temporally constituted perceptions within it but rather as a potentiality enacting temporal constitution and immanentisation through reflection, a delicate question to reorient the discussion is this: Can a transcendental event such as a time-constituting process be perceived by any other means except by (temporal) reflection? Isn't it necessarily a givenness in reflection? And what meaning can we bestow to the term reflection? Moreover, how could a non-temporally constituted process be given otherwise than by reflection? How could it draw phenomenological perception in the sense of a fulfillment of intentional experience without being the content of a consciousness intentionally 
directed upon it? Its phenomenological perception would then be inaccessible to reflection except by being in the form of a time-constituting process whose object would be the Original process. In Husserl's view though, this kind of process not only can be given in reflection in temporal form but there also exists evidence that this process is constituted as a process prior to any reflection, which means that being and beingin constituting within it are inseparable (Husserl (2001), p. 206).

Undoubtedly, the recourse to this transcendence within the immanence of consciousness in attempting to ground a temporal unity constituting subjectivity and at the same time to avoid an endless regression of reflective regards 'out of it' and 'towards it', being meant as consciousness-of something, bears in mind the description of the absolute ego of consciousness in Husserl's Phenomenology of Inner TimeConsciousness as an essentially atemporal subjectivity, root of the selfconstituting unity of the absolute flux of consciousness (Hussel (1966), $\S 36)$.

As a matter of fact, Husserl would not come closer even in his latest texts to elucidating the inherently vague concept of absolute ego of consciousness (or of any term used as equivalent to it). In my view, this is a manifestation of the transcendental and therefore impredicative root of all temporally constituted continuous unity of the Life-World, of all physical processes within it (involving the special presence of a bodily consciousness) reflected in their formal representation in metatheoretical logical-mathematical structures.

As a concluding remark to this article, the question of a continuous residuum of time in quantum-mechanical 'observation' (reflected also in the corresponding mathematical theory) taken as most closely associated with phenomenologically motivated lowest-level intentional 'observation', as well as relevant clues drawn from an empirical-experimental context, seem to lead to a deeper subjective root of the continuous unity of the broadly conceived epistemological edifice of the physical 
world. Even in rejecting any phenomenological connotations it is hard to argue that such a root would not be time-constituting.

\section{REFERENCES}

ANASTOPOULOS, C. "Continuous-time histories: Observables, probabilities, phase space structure and the classical limit". Jour. of Mathematical Physics, 42, 8, pp. 3225- 3259, 2001.

DEHAENE, S., SERGENT, C., \& BAILLET, S. "Timing of the brain events underlying access to consciousness during the attentional blink". Nature Neuroscience, 8, pp. 1391-1400, 2005.

DIRAC, P. The Principles of Quantum Mechanics. Oxford University Press, 1981.

di RUSSO, F., Mc DONALD, J., TEDER-SÄLERJÄRVI, W. , HILLYARD, S. "Neural basis of auditory-induced shifts in visual timeorder perception". Nature Neuroscience, 8, pp. 1197-1202, 2005.

ECCLES, J. Évolution du cerveau et création de la conscience, transl. Jean-Mathieu Luccioni. Paris: A. Fayard, 1992.

— . "The mind-brain problem revisited: the microsite hypothesis". In: J.C. Eccles \& O.D. Creutzfeldt (eds) (1989), p. 549.

ECCLES, J.; CREUTZFELDT, O.D. The Principles of Design and Operation of the Brain. Vatican City: Pontificiae Academiae Scientiarum Scripta Varia, 1989.

FARRUKH, O.M. "Application of nonstandard analysis to quantum mechanics". Journal of Mathematical Physics, 16, 2, pp. 177$200,1975$.

Manuscrito - Rev. Int. Fil., Campinas, v. 34, n. 2, pp. 435-469, jul.-dez. 2011. 
FRENCH, S. "Identity and Individuality in Classical and Quantum Physics". Australasian Journal of Philosophy, 67, 4, pp. 432446, 1989.

—_. "A phenomenological solution to the measurement problem? Husserl and the foundations of quantum mechanics". Studies in History and Philosophy of Modern Physics, 33, pp. 467-491, 2002 .

GELL-MANN, M. \& HARTLE, J. "Alternative decohering histories in quantum mechanics". In: K.K. Phua and Y. Yamaguchi (eds) (1990a).

- "Quantum mechanics in the light of quantum cosmology". In: S. Kobayashi et al. (eds) (1990b), pp. 321-343.

HARTLE, J. "Spacetime quantum mechanics and the quantum mechanics of spacetime". In: B. Julia, J. Zinn-Justin (eds) (1993), pp. $285-480$.

HANDY, T., GRAFTON, S., SCHROFF, N., KETAY, S., GAZZANIGA, M. "Graspable objects grab attention when the potential for action is recognized". Nature Neuroscience, 6, pp. 421-427, 2003.

HUSSERL, E. Ideen zu einer reinen Phänomenologie und phänomenologischen Philosophie. Erstes Buch, Husserliana, Band III/I, herausg. K. Schuhmann, Kluwer Acad. Pub., Dordrecht: The Netherlands, 1995.

_. Zur Phänomenologie des Inneren Zeitbewusstseins, herausg. R Boehm, Husserliana Band X, M. Nijhoff, Haag: The Netherlands, 1966.

—. Die Bernauer Manusckripte über das Zeitbewusstsein (1917/18), Husserliana Band XXXIII, herausg. R. Bernet \& D. Lohmar, Kluwer Acad. Pub., Dordrecht: The Netherlands, 2001. 
HUSSERL, E. Ideen zu einer reinen Phänomenologie und phänomenologischen Philosophie. Drittes Buch, Husserliana Band V, herausg. M. Biemel, Kluwer Acad. Pub., Dordrecht: The Netherlands, 1997.

. Formale und Transzendentale Logik, Husserliana Band XVII, herausg. P. Janssen, M. Nijhoff, Den Haag: The Netherlands, 1974 .

- Logische Untersuchungen, Ergänzungsband, Erster Teil, [Entwürfe zur Ummarbeitung der VI. Untersuchung and zur Vorrede für die Neuauflage der Logischen Untesuchungen (Sommer 1913)], Husserliana Band XX/I, herausg. Ul. Melle, Kluwer Acad. Pub., Dordrecht: The Netherlands, 2002.

- Logische Untersuchungen, (Erster Band: Prolegomena zur Reinen Logik), Husserliana Band XVIII, herausgb. E. Holenstein, M. Nijhoff, Den Haag: The Netherlands, 1975.

—. Späte Texte uber Zeitkonstitution (1929-1934): die C-Manuskripte, Husserliana: Materialienband VIII, herausgb. D. Lohmar, Springer, Dordrecht: The Netherlands, 2006.

ISHAM, J.C. "Quantum Logic and the Histories Approach to Quantum Theory". arXiv:gr-qc/9308006v19 Aug 1993, 1993.

ISHAM, J.C. \& LINDEN, N. "Quantum Temporal Logic and Decoherence Funcionals in the Histories Approach to Generalized Quantum Theory". arXiv:gr-qc/9405029v1 11 May 1994, 1994.

ISHAM, J.C. \& SAVVIDOU, N.K. "Time and Modern Physics". In K. Ridderbos (ed), Time, pp. 6-26. Cambridge: Cambridge University Press, 2002.

JULIA, B; ZINN-JUSTIN, J. (eds) Proceedings of the 1992 Les Houches School, Gravitation and Quantisation. Elsevier Science, 1995. 
KOBAYASHI, S.; EZAWA, H.; MURAYAMA, Y.; NOMURA, S (eds). Proceedings of the Third International Symposium on the Foundations of Quantum Mechanics in the Light of New Technology. Physical Society of Japan, Tokio, 1990.

LIVADAS, S. "The Phenomenological Roots of Nonstandard Mathematics". Romanian Jour. of Infor. Science and Technology, 8, 2, 115-136, 2005.

LORENZ, K. Behind the Mirror. London: Methuen, 1977.

PHUA, K.K.; YAMAGUCHI, Y. Proceedings of the 25th International Conference on High Energy physics, Singapore, August, 2-8, 1990. Singapore: World Scientific, 1990.

SAVVIDOU, N. "The action operator for continuous-time histories". Jour. of Mathematical Physics, 42, 11, pp. 5657-5674, 1999.

SOKOLOWSKI, R. Husserlian Meditations. Evanston: Northwestern University Press, 1974.

UTTAL, W.R. The Psychobiology of Mind. Hillsdale, NJ: Lawrence Erlbaum, 1978. 